\title{
Primary malignant melanoma of the uterine cervix treated with radical surgery and adjuvant chemo-radiation using temozolomide and cisplatin: a case report
}

\author{
Goter Doke $^{1 *}$, Shyam Tsering ${ }^{2}$, Hage Nobin³, Dacto Gara ${ }^{4}$
}

\begin{abstract}
${ }^{1}$ Department of Obstetrics and Gynecology, ${ }^{2}$ Department of Radiotherapy, ${ }^{3}$ Department of Pathology, ${ }^{4}$ Department of Surgical Oncology, TRIHMS, Naharlagun, Arunachal Pradesh, India
\end{abstract}

Received: 03 June 2019

Accepted: 17 July 2019

\author{
*Correspondence: \\ Dr. Goter Doke, \\ E-mail: dokegoter@gmail.com
}

Copyright: (c) the author(s), publisher and licensee Medip Academy. This is an open-access article distributed under the terms of the Creative Commons Attribution Non-Commercial License, which permits unrestricted non-commercial use, distribution, and reproduction in any medium, provided the original work is properly cited.

\begin{abstract}
Primary malignant melanoma of the uterine cervix is a rare neoplasm and the overall prognosis of patients with this disease is very poor. Herein, authors report a case of 45-year-old woman who presented with vaginal bleeding for one months and examination showed an exophytic, $6 \mathrm{~cm}$ polypoid blackish-pigmented tumor on the cervix involving vaginal fornix. She underwent abdominal radical hysterectomy with bilateral salpingo-oophorectomy and pelvic lymphadenectomy and further received adjuvant concurrent chemo-radiation with cisplatin (CDDP) and temozolamide but died 7 months after surgery.
\end{abstract}

Keywords: Cervix, Cisplatin, Primary malignant melanoma, Temozolamide

\section{INTRODUCTION}

Melanocytes were first demonstrated in the cervical epithelium in 1959 and since then malignant melanoma of cervix has been reported as separate entity. ${ }^{1}$ Approximately $3 \%$ to $7 \%$ of malignant melanoma in women develops within genital tract. Primary malignant melanoma of uterine cervix is extremely rare with incidence five times lower than vagina and vulva cases. ${ }^{2,3}$

Presently, there is no consensus regarding standard treatment of primary malignant melanoma of cervix and all the published literature has reported a very poor longterm survival..$^{3-5}$ Authors treated the patient with adjuvant chemo-radiation using temozolomide and cisplatin after radical surgery. To this knowledge, there are very few studies reported on use of temozolomide and cisplatin as adjuvant chemotherapy in primary malignant melanoma of cervix.

\section{CASE REPORT}

A 45-year-old patient presented with vaginal bleeding for 1-months. Gynecologic examination revealed a $6 \mathrm{~cm}$ polypoid blackish-pigmented tumor in the cervix with involvement of the upper vaginal fornix (Figure 1). A punch biopsy and the histological examination revealed the existence of a malignant melanoma cell in cervix and immunohistochemistry showed positive reaction for vimentin, melan- A and S-100. Patient underwent an extensive search for melanotic lesion of skin or other sites, which was negative. The clinical diagnosis made for this patient was International Federation of Gynecology and Obstetrics (FIGO) stage II A2.

On CECT imaging, a lesion $6 \times 4 \times 4 \mathrm{~cm}$ occupying the cervix was identified with involvement of lower uterus. Additionally, a $12 \mathrm{~mm}$ equivocal right internal iliac lymph node was noted. But there was no evidence of 
extra-cervical disease on a CT scan of chest and abdomen.

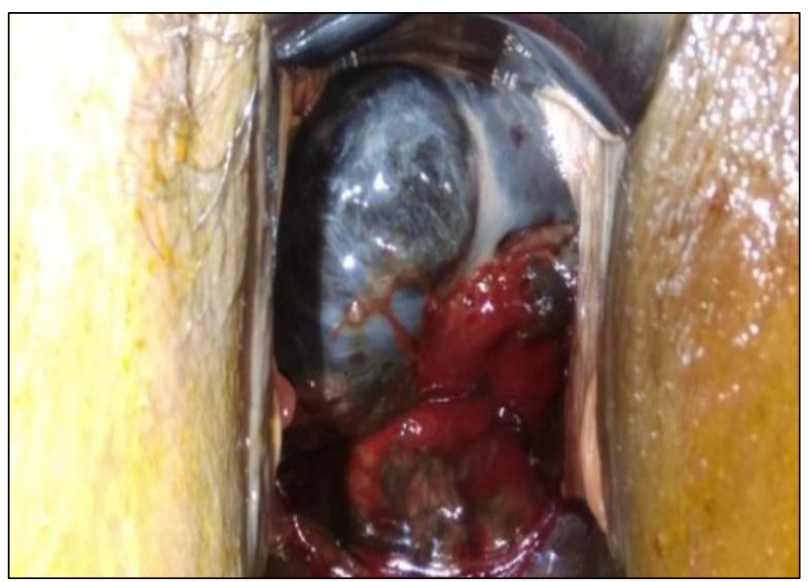

Figure 1: Macroscopic findings demonstrated a $6 \mathrm{~cm}$ polypoid blackish-pigmented tumor in the cervix and involving the lower third of the vagina.

Considering the poor prognosis of malignant melanoma, this multidisciplinary groups decided to take aggressive treatments consisting of radical hysterectomy and adjuvant concurrent chemo-radiation. The patient had the Eastern Cooperative Oncology Group (ECOG) performance status 1 and she underwent Type III Radical Abdominal Hysterectomy with bilateral salpingooophorectomy and bilateral pelvic lymphadenectomy without any major complications (Figure 2).

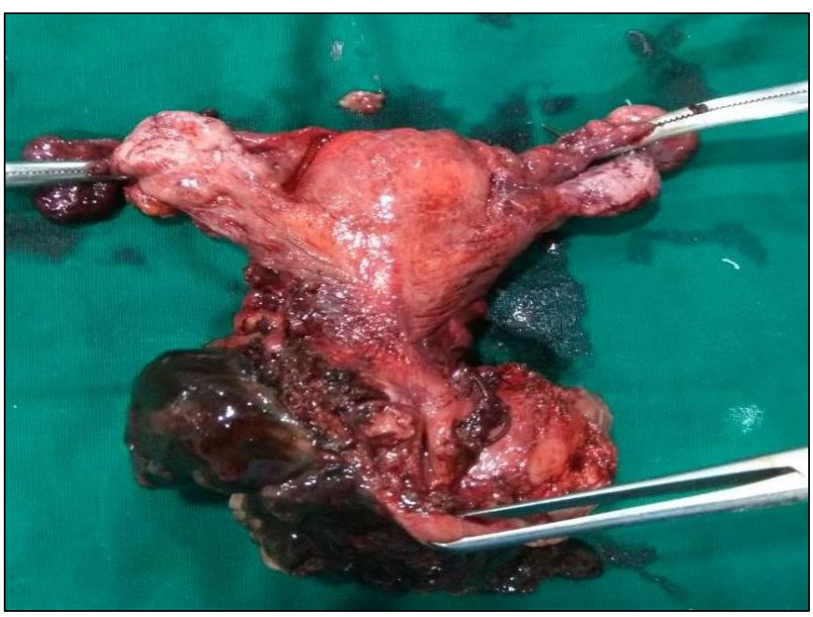

Figure 2: Surgical specimens showed an enlarged uterus with a huge dark black ulcer fungating tumor arising in the cervix, extending into the vagina with full-thickness invasion of the cervical wall.

The final histopathological report showed a malignant melanoma of cervix with tumor size $7.5 \times 4.5 \times 4.5 \mathrm{~cm}$. The tumor composed of large pleomorphic polygonal oval to spindle cells with intracytoplasmic melanin deposits of mitotic count up to 12 per 10 high-powered fields (Figure 3). The vaginal resection margin, bilateral parametrium and 8 out of 26 pelvic lymphnodes were found to be involved. Therefore, the final histopathological diagnosis made was stage III B primary cervical malignant melanoma according to the International Federation of Gynecology and Obstetrics staging system. As planned, six cycles of intravenous cisplatin (CDDP) $20 \mathrm{mg} / \mathrm{m} 2$ and oral temozolomide $200 \mathrm{mg} / \mathrm{m} 2$ were administered at every 3 weeks interval and concurrently, whole pelvis was treated with daily 200 cGy in 25 fractions by Box technique up to total of 5,000 cGy with external beam radiation therapy, followed by 350 cGy with high dose rate intracavitary brachytherapy. The patient tolerated and completed the adjuvant treatment and was sent home with follow up advices.

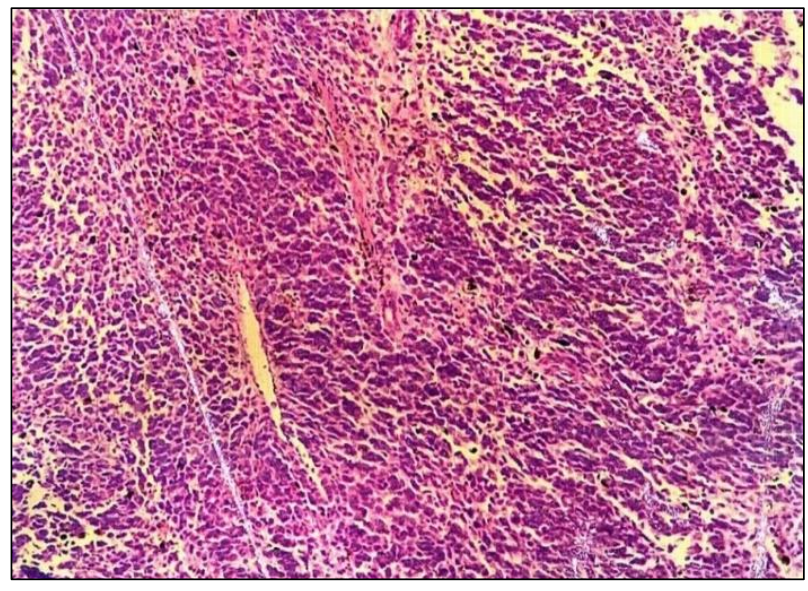

Figure 3: Histo-pathological findings of the cervix revealed diffuse infiltration of large pleomorphic polygonal oval to spindle cells with prominent eosinophilic nucleoli and marked pigment deposits and abundant blood vessel. (H\&E, ×400).

But she presented again with a palpable abdominal mass and dyspnoea on her first follow up at 3-months. Local vaginal examination was normal but CECT scan showed a large $8 \times 8 \mathrm{~cm}$ right iliac fossa mass encasing the right ureter causing hydro-nephrosis and multiple lung metastases. She and her family opted for palliative care, and she died 7 months after surgery.

\section{DISCUSSION}

Primary malignant melanoma of the cervix is very rare and have been reported in age group from 19 to 83 years with peak incidence between 60 and 70 years, and it is likely to present with vaginal discharge, bleeding or dyspareunia. ${ }^{3,5}$ The age of the present case was 45 years and vaginal bleeding was the clinical presentation, which was similar with the previous studies. The diagnosis is usually based on gynecologic examination, colposcopy, and cervical pathology. Pap smears usually shows round or spindle atypical cells containing melanin pigments. ${ }^{6}$ About half of the melanomas are amelanotic, and the diagnosis of amelanotic melanoma may be difficult to distinguish from sarcomas and poorly differentiated 
squamous cell carcinoma. Immunostaining is useful for the diagnosis of MM. Protein $\mathrm{S} 100$ is considered sensitive and protein HMB 45 more specific, and it is more useful when the two markers are combined. ${ }^{6}$ In authors patient vimentin, Melan-A and S-100 was positive thus supporting the diagnosis of malignant melanoma. Norris and Taylor proposed four criteria for the diagnosis of primary cervical MM: (i) presence of melanin in the normal cervical epithelium, (ii) absence of melanoma elsewhere in the body, (iii) demonstration of junctional change in the cervix, and (iv) metastases according to the pattern of cervical carcinoma. ${ }^{7}$ In this case, the tumor size of the cervix was larger, and the invasion depth of the cervical tumor was deeper than that of the vaginal tumors. Therefore, authors conclude that the cervix was the primary site and it disseminated to the vaginal wall.

While there is no consensus regarding the standard management of cervical MM, surgery remains the major therapeutic method. ${ }^{3,5,8}$ Some authors advocates radical hysterectomy to obtain clean surgical margins of at least $2 \mathrm{~cm}^{4,9}$ Other researchers suggested that a more conservative surgical approach may be appropriate considering poor prognosis. ${ }^{10,11}$ Also considering the metastases pattern of primary Malignant Melanoma of cervix as similar to cervical carcinoma, 7 radical hysterectomy has been advocated to improve the prognosis. In the present case, type III radical hysterectomy with bilateral salphingoopherectomy and bilateral pelvic lymphadenectomy was done. However, the histopathology showed vaginal resection margin, both parametrium and pelvic lymphnodes to be involved, which warranted the need of adjuvant chemo-radiation.

Presently, there is no chemotherapeutic regimen which has valid effect on malignant melanoma. Chemotherapy are performed using the same protocol for skin melanoma. ${ }^{8,12}$ Dacarbazine is the most widely used drug with $15-20 \%$ response rates. Combination chemotherapy with cisplatin, vinblastine, and dacarbazine led to RR of 20-35\%, but this was not more effective than dacarbazine alone for prolonging survival. ${ }^{13}$ Temozolomide (TMZ), an oral imidazotetrazine has been used in advanced melanoma and primary brain tumors. TMZ and dacarbazine share the active intermediary 5-(3methyltriazen-1-yl) imidazole-4-carboximide (MTIC). However, unlike dacarbazine, which requires metabolic activation, TMZ spontaneously converts to MTIC under physiological conditions. It has high oral bioavailability and extensive tissue distribution, including penetration through the blood-brain barrier. ${ }^{14}$ A randomized phase III trial showed equivalent overall survival and toxicity with increased progression-free survival and better quality of life with TMZ than Dacarbazine. ${ }^{15}$

A randomized phase II study comparing combination of temozolomide and cisplatin vs temozolomide alone in patients with advanced melanoma reported no superiority in terms of response rate, median TTP or OS using combination agent. However, this study justified the use of temozolomide in patients with brain metastases. ${ }^{14}$ Thus, combination of TMZ and cisplatin was also used in this case as adjuvant chemotherapy agent along with radiation to improve the outcome. Recently, new therapies targeted against BRAF mutations (Dabrafenib), c-KIT mutations (Imatinib), inhibitor of MEK1 and MEK2 (Trametinib) and monoclonal antibody (Ipilimumab) have been reported to have good results in advanced stage melanoma. ${ }^{16}$ Only one case has been reported on use of pembrolizumab in primary MM of uterine cervix but showed very poor response. ${ }^{17}$ Malignant Melanoma is considered a radio-resistant tumor and radiotherapy has been used as an adjuvant and palliative treatment in some case report. ${ }^{8}$ Adjuvant pelvic RT has been applied to patients with unsatisfactory surgical resection, infiltrated parametriums and involved lymphnodes. ${ }^{12,18}$ In the present case, adjuvant radiation was used because of the same findings. Unquestionably, tumor stage and tumor thickness represent the main prognostic factor of this rare disease. Pusceddu et al, reported that only $10.7 \%$ of patients lived more than 5 years while majority of patients died within 3 years $(87.5 \%)$ and 5-year survival rate observed were $18.8 \%$ for stage I, $11.1 \%$ for stage II and $0 \%$ for stages III-IV. ${ }^{3}$ Recent review also reported that $50.0 \%$ of patients survived for more than two years, and only two patients $(14.3 \%)$ survived for more than five years. ${ }^{5}$ In this case, patient survived only for 7 months after undergoing surgical treatment and chemo-radiation.

\section{CONCLUSION}

Further studies are needed in order to propose standard treatment for primary malignant melanoma of cervix.

Funding: No funding sources

Conflict of interest: None declared

Ethical approval: Not required

\section{REFERENCES}

1. Cid JM. Melanoid pigmentation of the endocervix: a neurogenic visceral argument. Ann Anat Pathol (Paris). 1959;4:617-28.

2. Patrick RJ, Fenske NA, Messina JL. Primary mucosal melanoma. J Am Acad Dermatol. 2007;56(5):828-34.

3. Pusceddu S, Bajetta E, Carcangiu ML, Formisano B, Ducceschi M, Buzzoni R. A literature overview of primary cervical malignant melanoma: an exceedingly rare cancer. Crit Rev Oncol Hematol. 2011;81(2):103-206.

4. Cantuaria G, Angioli R, Nahmias J, Estape R, Penalver M. Primary malignant melanoma of the uterine cervix: case report and review of the literature. Gynecol Oncol. 1999;75(1):170-4.

5. Yuan $\mathrm{G}, \mathrm{Wu} \mathrm{L}, \mathrm{Li} \mathrm{B}, \mathrm{An}$ J. Primary malignant melanoma of the cervix: report of 14 cases and review of literature. Oncotarget. 2017;8(42):73162-7. 
6. Deshpande AH, Munshi MM. Primary malignant melanoma of the uterine cervix: report of a case diagnosed by cervical scrape cytology and review of the literature. Diagn Cytopathol. 2001;25(2):108-11.

7. Norris HJ, Taylor HB. Melanomas of the vagina. Am J Clin Pathol. 1966;46(4):420-6.

8. Sugiyama VE, Chan JK, Kapp DS. Management of melanomas of the female genital tract. Curr Opin Oncol. 2008;20(5):565-9.

9. Wydra D, Sawicki S, Ciach K, Emerich J. Malignant melanoma of the uterine cervix. Eur $\mathrm{J}$ Obstet Gynecol Reprod Biol. 2006;124(2):257-8.

10. Kristiansen SB, Anderson R, Cohen DM. Primary malignant melanoma of the cervix and review of the literature. Gynecol Oncol. 1992;47(3):398-403.

11. Santoso JT, Kucera PR, Ray J. Primary malignant melanoma of the uterine cervix: two case reports and a century's review. Obstet Gynecol Surv. 1990;45(11):733-40.

12. Piura B. Management of primary melanoma of the female urogenital tract. Lancet Oncol. 2008;9(10):973-81.

13. Bajetta E, Del Vecchio M, Nova P, Fusi A, Daponte A, Sertoli MR, et al. Multicenter phase III randomized trial of polychemotherapy (CVD regimen) versus the same chemotherapy (CT) plus subcutaneous interleukin- 2 and interferon-alpha2b in metastatic melanoma. Ann Oncol. 2006;17(4):571-7.

14. Bafaloukos D, Tsoutsos D, Kalofonos H, Chalkidou S, Panagiotou P, Linardou E, et al. Temozolomide and cisplatin versus temozolomide in patients with advanced melanoma: A randomized phase II study of the Hellenic Cooperative Oncology Group. Ann Oncol. 2005;16(6):950-7.

15. Middleton MR, Grob JJ, Aaronson N, Fierlbeck G, Tilgen W, Seiter S, et al. Randomized phase III study of temozolomide versus dacarbazine in the treatment of patients with advanced metastatic melanoma. J Clin Oncol. 2000;18(1):158-66.

16. Van Zeijl MC, van den Eertwegh AJ, Haanen JB, Wouters MW. (Neo)adjuvant systemic therapy for melanoma. Eur J Surg Oncol. 2017;43(3):534-43.

17. Kim MS, Choi C, Kim T, Lee J, Lee J, Bae D. Primary malignant melanoma of the uterine cervix treated with pembrolizumab after radical surgery: a case report and literature review. Obstet Gynecol Sci. 2018;61(4):524-8.

18. Mousavi AS, Fakor F, Nazari Z, Ghaemmaghami F, Hashemi FA, Jamali M. Primary malignant melanoma of the uterine cervix: case report and review of the literature. J Low Genit Tract Dis. 2006;10(4):258-63.

Cite this article as: Doke G, Tsering S, Nobin H, Gara D. Primary malignant melanoma of the uterine cervix treated with radical surgery and adjuvant chemo-radiation using temozolomide and cisplatin: a case report. Int J Reprod Contracept Obstet Gynecol 2019;8:3387-90. 